\title{
Erratum to: A Probability Approach to Anomaly Detection with Twin Support Vector Machines
}

\author{
$N I E W e i^{*}$ (聂 巍), HE Di (何 迪) \\ (Department of Electronic Engineering, Shanghai Jiaotong University, Shanghai 200240, China) \\ (C) Shanghai Jiaotong University and Springer-Verlag Berlin Heidelberg 2010
}

In the original version of the article, the Foundation item contains a mistake. The correct Foundation item should be: the National Natural Science Foundation of China (No.60802058) and the Scientific Research Foundation for the Returned Overseas Chinese Scholars, State Education Ministry.

\footnotetext{
The online version of the original article can be found at http://dx.doi.org/10.1007/s12204-010-1021-3.
}

\footnotetext{
*E-mail: nie.wei@gateh.edu
} 